\title{
Postmortem diagnosis of hypothermia
}

\author{
Cristian Palmiere • Grzegorz Teresiński • Petr Hejna
}

Received: 11 November 2013 / Accepted: 4 February 2014 / Published online: 21 February 2014

(C) Springer-Verlag Berlin Heidelberg 2014

\begin{abstract}
The identification of hypothermia as the cause of death has always been somewhat problematic in forensic pathology because of unspecific, inconstant, or even negative macroscopic and microscopic findings. Though the simultaneous presence of frost erythema, Wischnewski spots, hemorrhages into the synovial membrane, bloody discoloration of synovial fluid of the knee, and basal vacuolization of the renal tubular epithelial cells has been indicated as strongly supportive of fatal hypothermia, their absence does not allow the diagnosis of hypothermia to be ruled out. Postmortem biochemical investigations are valuable in detecting adaptation responses to cold stress and metabolic changes that occur following cold exposure. However, ethanol intoxication prevents appearance of adaptation responses to cold, rendering the diagnosis less obvious. Immunohistochemistry, postmortem imaging, and molecular pathology have shown promising results, although at present, they do not provide pathognomonic signs of fatal hypothermia. The aim of this article is to present a review of the literature covering the significance of different postmortem investigations that are associated with hypothermia fatalities.
\end{abstract}

\footnotetext{
C. Palmiere $(\bowtie)$

University Centre of Legal Medicine, Lausanne University Hospital, Rue du Bugnon 21, 1011 Lausanne, Switzerland

e-mail: cristian.palmiere@chuv.ch

G. Teresiński

Department of Forensic Medicine, Medical University of Lublin, Lublin, Poland

P. Hejna

Department of Forensic Medicine, Charles University in Prague, Faculty of Medicine and University Hospital Hradec Králové, Sokolská 581, 50005 Hradec Králové, Czech Republic
}

Keywords Hypothermia · Forensic pathology · Autopsy · Histology $\cdot$ Postmortem biochemistry $\cdot$ Postmortem imaging

\section{Introduction}

Hypothermia occurs when the core body temperature is $35^{\circ} \mathrm{C}$ or less, approximately $2{ }^{\circ} \mathrm{C}$ below the normal body temperature. In clinical terms, hypothermia is classified according to severity as mild, moderate, and severe. Mild hypothermia is usually defined as $35-32{ }^{\circ} \mathrm{C}$, moderate as $32-28{ }^{\circ} \mathrm{C}$, and severe as $<28{ }^{\circ} \mathrm{C}[1]$.

Hypothermia from environmental exposure to cold (primary hypothermia) appears when the body's heat production and heat retention mechanisms fail to prevent continuous heat loss. By contrast, secondary hypothermia occurs in the context of underlying clinical conditions or concurrent medications that affect the body's ability to maintain its internal core temperature (e.g., hypothalamic diseases or medications that impair central thermoregulation). Exposure to low temperatures may result in focal (localized hypothermia), systemic (general hypothermia) injury and, possibly, death.

The risk of death by hypothermia is particularly high during prolonged exposure to subfreezing environmental temperatures $\left(0^{\circ} \mathrm{C}\right)$, though fatal hypothermia may also be encountered at higher environmental temperatures. Not surprisingly, both the very young and the elderly have been identified as being especially susceptible to fatal cold exposure. Compared to adults, infants have an increased body surface area and decreased body mass index in conjunction with underdeveloped thermoregulatory mechanisms.

In the aged, the risk of hypothermia may be enhanced by reduced heat production, increased heat loss and impaired thermoregulation. These may the consequence of physiologic decreases in subcutaneous fat and muscle, chronic diseases, 
undernourishment, primary or secondary disorders of the central nervous system and inactivity related to senescence.

Although fatal cases of hypothermia usually occur following exposure to extreme environmental temperatures, deaths may also occur indoors. An elderly person, for instance, suffering from preexisting, debilitating conditions may become hypothermic even at temperatures as high as $22-24{ }^{\circ} \mathrm{C}$ $[2,3]$.

Other cases particularly vulnerable to the development of fatal hypothermia include the homeless, the chronically ill, the isolated or socially deprived individuals, and those suffering from psychiatric diseases as well as drug or alcohol intoxication.

As far as the last is concerned, ethanol is the most commonly detected drug in the blood of victims succumbing to primary hypothermia. This is not surprising for a myriad of reasons. First, ethanol can accelerate body heat loss through continuous peripheral vasodilatation, thereby leading to a false feeling of warmth that may also inhibit heat production through shivering. Additionally, its intoxicating effects impair appropriate decision-making in cold environments and disturb the thermoregulatory set point by acting on the hypothalamus. Furthermore, ethanol-induced shifts in the hepatocyte redox potential influence biochemical responses to cold, e.g., ketogenesis and gluconeogenesis. Many chronic alcoholics are also malnourished and thus more prone to hypothermia [4].

Some pharmacologic agents can cause central thermoregulatory failure (e.g., paracetamol, barbiturates, opioids, tricyclic antidepressants, and benzodiazepines). Phenothiazines can both impair central thermoregulation and inhibit peripheral vasoconstriction in response to cold through their $\alpha$ blocking activity. Other $\alpha$-blockers, such as prazosin, have been reported to cause hypothermia, the elderly being particularly susceptible to this effect. Valproic acid has also been reported to cause hypothermia in a handful of cases with a mechanism possibly related to the $\gamma$-aminobutyric acid agonistic effect $[2,5,6]$.

In the forensic setting, hypothermia may occur on land (dry hypothermia) or in water (wet or immersion hypothermia). Based on pathophysiological changes, hypothermia can also be classified as acute, subacute, or chronic. Acute hypothermia occurs most commonly following immersion in cold water. In these situations, intense cold stress may overwhelm heat production so that the body cools before its energy reserves are exhausted. Indeed, the thermal conductivity of water is 20 times that of air. In addition, water aspiration may be responsible for or contribute to a fatal outcome, the cause of death then being drowning rather than hypothermia. Immersion in cold water may also precipitate vagal reflexes, possibly leading to sudden cardiac arrest. In subacute hypothermia, the cold is less severe and cooling only occurs when energy reserves are exhausted. In chronic hypothermia, victims are exposed to moderate cold for days. Such situations can be typically encountered by the elderly living in poor housing or by those with malnutrition and debilitating diseases, as previously described $[7,8]$.

\section{Macroscopic findings}

The identification of hypothermia as the cause of death has always been somewhat problematic in forensic pathology due to unspecific, inconsistent, or even negative autopsy findings. The macroscopic changes with the highest diagnostic validity were described by Keferstein (frost erythema), Wischnewski (hemorrhagic spots of the gastric mucosa), and Krjukoff at the end of the nineteenth century and beginning of the twentieth century. Although extensive research carried out over the years has aimed to define more reliable criteria, significant advances in diagnosing hypothermia as the cause of death have been relatively limited [7, 9].

In 1976, Hirvonen [10] concluded that "in fatalities due to hypothermia the necropsy findings event at their best can be scarce. There are cases where no morphological signs are noticed and the diagnosis must be based solely on circumstantial evidence. Textbooks of forensic sciences and other sources report cadaveric signs such as red livores, purple patches on extremities, red blood, Wischnewski erosions in the stomach, pancreatic haemorrhage, lipid depletion from the adrenal cortex and even vacuolization of the liver cells and other similar minute signs of hypoxia."

Similar conclusions were reported by Coe [11] in 1984, who emphasized that "death by hypothermia is very difficult to verify, because there are no diagnostic autopsy findings. Gross lesions that have been observed include swollen, discolored extremities, ears, and nose; hemorrhage or petechial ulcers of the gastric mucosa; and hemorrhagic pancreatitis. Microscopic foci of myocardial degeneration have been described. The skin changes are suggestive but not diagnostic of frostbite, and all the internal findings, both gross and microscopic, are manifestation of stress that may be found in other conditions than hypothermia. As a consequence, the diagnosis of death by hypothermia is made by a combination of observations, including an appropriate history of exposure, certain nonspecific pathological finds when present, and the absence of other lethal factors."

There are no pathognomonic signs of fatal hypothermia at autopsy. Furthermore, morphological changes are generally unspecific and do not allow unambiguous diagnoses of death from hypothermia to be reached. However, there is a general consensus in the literature regarding hemorrhagic spots of the gastric mucosa (Wischnewski spots) in conjunction with reddish brown skin discoloration over the extensor surfaces of the large joints (frost/cold erythema) as "classical signs" or as "highly indicative" or "supportive" of fatal hypothermia. 
Hemorrhages of the synovial membrane, bloody discoloration of the synovial fluid, bright red or pink lividity, pancreatic hemorrhages, hemorrhages into the large muscles of the body, especially the iliopsoas muscle, as well as hemorrhagic spots in the duodenum and jejunum have also been reported in association with fatal hypothermia, albeit less frequently. All these findings must, nevertheless, be correlated with the circumstances from the death scene [7, 9, 12-14].

Hemorrhagic spots of the gastric mucosa (Wischnewski spots) are disseminated, dark lesions of the gastric mucosa that may be associated with fatal hypothermia. Similar findings may also be found, though less commonly, in the lower gastrointestinal tract and, rarely, in the esophagus or regions with ectopic gastric mucosa. Wischnewski spots may vary in size and quantity from $1 \mathrm{~mm}$ up to about $2 \mathrm{~cm}$ in diameter and from only a few up to more than 100 in number. Their incidence in fatal hypothermia has been reported in literature to vary between 40 and $100 \%$. Such a wide variation in the occurrence of Wischnewski spots may likely be due to the lack of diagnostic homogeneity in the algorithms used for postmortem confirmation of hypothermia death [7, 9, 14, 15].

Tsokos et al. [14] postulated that Wischnewski spots do not represent true erosions or ulcerations in terms of histopathologic diagnosis, even though they are frequently termed "gastric erosions" or "ulcerations" or "erosive gastritis." These authors observed that neither the lesions nor the adjacent gastric mucosa is characterized by inflammatory infiltrates or fibrin exudates. In order to explain their pathogenesis, Tsokos et al. formulated the hypothesis that the body's cooling in cold environmental conditions may primarily lead to discrete, circumscribed hemorrhages of the gastric glands in vivo or during the agonal period. Owing to subsequent erythrocyte autolysis, the released hemoglobin would be hematinized by gastric acid, thereby leading to the typical blackish-brownish appearance of Wischnewski spots at postmortem examination. Despite the convincing arguments proposed by some authors, both the morphology and pathogenesis of Wischnewski spots remain contentious. Their sensitivity and specificity as markers of hypothermia have therefore not been clearly determined. Moreover, while the absence of Wischnewski spots does not exclude the diagnosis of hypothermia, their presence does not necessarily indicate it. Indeed, a range of factors, beyond the simple lowering of body temperature, may influence Wischnewski spots formation.

Frost erythema forms reddish purple to violet or brownish areas of skin discoloration that are most commonly located over the extensor surfaces of the large joints, typically the knees, elbows, and great trochanter region. Frost erythema can also be observed on projecting areas of the face (ears, nose, and zygomatic areas), over the shoulders, and, in rare cases, on the male external genitalia. Discolorations of exposed skin areas have been reported to occur in up to $72 \%$ of cases of fatal dry hypothermia. However, they have not been observed following wet-cold exposure and must not be mistaken for hematomas. Microscopically, frost erythema is not characterized by erythrocyte extravasation and infiltration, although hemoglobin can be immunohistochemically visualized within the lesion, possibly originating from damaged erythrocytes due to cold hemolysis. Frost erythema is explained by cold hemolysis in superficial vessels running under the skin just above protruding bony surfaces (knee cap, olecranon, zygomatic bones, i.e., areas with rather thin subcutaneous fat tissue coverings) as superficially located bones are cooled more rapidly in the conduction mechanism. Frost erythema should not be mistaken for hemolytic pseudo-marbling staining of the skin that can sometimes appear alongside subcutaneous vessels after thawing of a frozen body. Skin incision and histologic sampling of suspected frost erythema areas are therefore highly recommended $[7,9,16]$.

Classic frostbites developing concomitantly with fatal hypothermia usually reveal edema and hyperemia without skin blistering or inflammation, the telltale signs of vital reaction in thawed survivors [17].

Hemorrhages of the synovial membrane and bloody discoloration of synovial fluid ("inner knee sign") have recently been proven to be reliable, vital signs for the diagnosis of death caused by hypothermia, with a diagnostic value comparable to that of frost erythema. Even though the pathogenesis of these findings is not entirely understood at present, their appearance might be related to the particularity of the synovial membrane's vascular supply as well as to the phenomena of erythrocyte congestion and extravasation into the synovial space within the synovial membrane following cold exposure. Similar to other morphologic signs suggesting hypothermia, the absence of these findings does not, however, exclude the diagnosis, and their appearance may be influenced by other factors. This is especially true regarding the presence of simultaneous antemortem trauma to the knees preceding death as well as other possible postmortem influences [12].

Bright red or pink lividity is of no diagnostic value since it may be found not only in hypothermia fatalities but also in many other situations characterized by exposure of the corpse to low environmental temperatures. Moreover, the phenomenon can be artificially reproduced by keeping the corpse in a cold environment $[7,9,12,16]$.

A large variety of pancreatic changes has been mentioned in the literature over the years, including local and diffuse hemorrhages and hemorrhagic pancreatitis $[10,13]$. According to Preuss et al. [13], who examined 143 cases of fatal hypothermia, bleeding on a macroscopic or microscopic level and signs of pancreatic inflammation can be ruled out as diagnostic criteria for death due to hypothermia. Most of the pancreatic changes traditionally associated with death by hypothermia might more likely be the expression of preexisting pancreatic diseases or the result of autolysis occurring with the onset of decompositional changes. 
Hemorrhages into the skeletal, paravertebral muscles, e.g., the iliopsoas muscles, have been reported to be associated with death by hypothermia, though not systematically. Skeletal muscle hemorrhages in hypothermia death were firstly mentioned in the classic German textbook of forensic medicine by von Hofmann and Haberda as early as 1927. Aside from the hypothesis concerning the increased capillary permeability resulting from hypoxic damage during hypothermia, the mechanisms responsible for their formation were not explored in more detail and their appearance remained a minor matter of debate in the literature [7,9]. Ogata et al. [18] reported a case of fatal hypothermia characterized by hemorrhages in the pectoralis minor, first intercostal, and iliopsoas muscles in the absence of skin injury or damage to the subcutaneous fat tissues, muscles, internal organs, or bones elsewhere. These investigators ruled out the possibility that these hemorrhages were the consequence of antemortem mechanical injuries or positional bleeding that occurred after death. They postulated that extremely intense shivering following cold exposure might be responsible for muscular hemorrhages. Furthermore, the intense effort of ventilation that occurs with shivering could contribute to bleeding into the pectoralis minor and intercostal muscles. Similarly, Simon's bleedings (stripe-like hemorrhages on the ventral surface of the intervertebral disks most commonly seen in the lumbar part of the spinal column) have also been occasionally observed in autopsy cases of fatal hypothermia, a possible consequence of intensive shivering [19].

Macroscopic findings that have been episodically or exceptionally described in association with fatal hypothermia include acute hemorrhagic necrosis of the esophagus ("black esophagus," possibly due to vasoconstriction in the splanchnic region), ulceration in the colon and ileum, as well as hemorrhagic infarctions of the colon. The latter was reported following hemodynamic and rheologic alterations characterizing hypothermia, with subsequent thromboses in the submucosal veins $[9,20]$. Hypothermia-related circulation disorders were found to be responsible for portal vein thrombosis in a report by Wolf et al. [21].

\section{Paradoxical undressing and hide-and-die syndrome}

Two peculiar aspects to some hypothermia cases are worth mentioning: paradoxical undressing and the "hide-and-die syndrome." Paradoxical undressing is a phenomenon characterized by the fact that victims, despite low environmental temperatures, paradoxically remove their clothes due to a sudden feeling of warmth. Clothes are often found close to the corpse. Paradoxical undressing may occur when body temperature drops to $29{ }^{\circ} \mathrm{C}$ and has been reported to occur both in children and adults. This irrational behavior has been explained by terminal changes in peripheral vascular resistance occurring in profoundly hypothermic individuals, possibly associated with diminished mental capacity due to advancing central cooling. Paradoxical undressing might represent a final effort on behalf of the victim, immediately followed by loss of consciousness and death.

The so-called hide-and-die syndrome or terminal burrowing behavior may also be observed in some cases of fatal hypothermia. The corpse is generally found in a hidden position, located under or behind furniture or other objects, reproducing a primitive, burrowing-like behavior of protection, as seen in hibernating animals. This phenomenon has mostly been observed in elderly persons but may occur in younger victims as well. Some authors have described corpses found under piles of books pulled from bookcases, hidden under a mound of clothes and newspapers, or burrowed under vegetation. It has been suggested that the hide-and-die syndrome may be either the result of mental confusion or a terminal, primitive reaction pattern, i.e., an autonomous behavior triggered by the brain stem in the final state of hypothermia. Care must be taken to evaluate the above scenario, especially in the concomitant presence of undressing found at the scene, as these signs might otherwise suggest crime and sexual assault $[3,7,15,22]$.

\section{Microscopic findings}

Several histologic findings associated with hypothermia fatalities have been described in medicolegal literature including depletion of hepatic glycogen, fatty degeneration of the renal tubular epithelium (subnuclear vacuolization of renal tubular epithelial cells or Armanni-Ebstein phenomenon), pancreatic changes (focal necrosis of pancreatic and peripancreatic adipose tissue, hemorrhagic and nonhemorrhagic pancreatitis, adenocyte vacuolation), vacuolization of anterior pituitary gland cells, hepatocytes, adrenal cells and renal cells, hypoxic cardiac changes as well as fatty changes of cardiac myocytes and hepatocytes. A strong correlation among frost erythema, Wischnewski spots, and basal vacuolization of renal tubular epithelial cells has been observed by some authors who postulated that the Armanni-Ebstein phenomenon might be considered the most "indicative" histological finding of hypothermia fatalities [7, 9, 13, 23-28].

Several groups of researchers have performed immunohistochemical investigations focusing on adrenocorticotropic hormone (ACTH), thyroid-stimulating hormone, noradrenaline, dopamine, chromogranin A, ubiquitin, myoglobin, heat shock protein 70 , microtubule-associated protein 2 , basic fibroblast growth factor, glial fibrillary acidic protein, $\mathrm{S} 100 \beta$, single-stranded DNA, pulmonary surfactant-associated proteins $\mathrm{A}$ and $\mathrm{D}$, atrial and brain natriuretic peptides, matrix metalloproteinase-2, matrix metalloproteinase-9, intercellular adhesion molecule-1, claudin-5, clusterin, and aquaporin 
immunoreactivities in various organs and tissues. These include the lungs, myocardium, anterior pituitary gland, adrenal medulla, hypothalamus, midbrain periaqueductal gray matter, renal tubular epithelium, distal and collecting renal tubules, glomerular podocytes, brain parietal lobe, hippocampus, and cerebral cortex, with extremely promising results in some cases. However, immunohistochemistry has still not provided significantly reliable diagnostic criteria for the postmortem diagnosis of hypothermia [24, 28-33].

\section{Postmortem biochemistry}

In recent years, there has been a significant revival, expansion, and development in postmortem biochemical investigations regarding hypothermia fatalities. The first reports concerning biochemistry in hypothermia in the forensic setting were published by Mant and focused on vitreous magnesium values. Since then, numerous molecules have been measured, and postmortem samples other than blood have been collected and analyzed in order to better understand the metabolic and biochemical changes occurring during hypothermia and find the most helpful combination of biomarkers to allow a reliable diagnosis of fatal hypothermia to be made. In addition, these investigations aimed to identify alternative biological fluids should blood prove insufficient or unavailable at autopsy [24].

Exposure to cold is characterized by significant stress reactions that enhance catecholamine and counter-regulatory hormone release. Enhanced fat catabolism and increased acetoacetate/ $\beta$-hydroxybutyrate production are the metabolic consequences of hypothermia-induced secretion of insulin antagonist hormones [34-38].

Recent observations have revealed that ketone production during controlled, intraoperative cardiac hypothermia required a much lower core temperature than the $34-35^{\circ} \mathrm{C}$ used in coronary artery bypass/valve replacement surgery since increased blood ketone levels were observed exclusively in those cooled to $16{ }^{\circ} \mathrm{C}$ during aortic aneurysm surgery [36].

There is a general consensus in the literature that increased levels of blood $\beta$-hydroxybutyrate, acetone, isopropyl alcohol, and glucocorticosteroids as well as increased urine adrenaline and cortisol concentrations should be considered potential biochemical hallmarks of fatal hypothermia [24, 34-37].

Indeed, $\beta$-hydroxybutyrate and acetone should be systematically determined in the blood of all suspected hypothermia cases as should levels of isopropyl alcohol. The latter may appear higher in blood in hypothermia fatalities due to increased acetone concentrations and subsequent enhanced acetone metabolism (if not of congeneric origin as a fermentation impurity of consumed ethanol). Blood $\beta$-hydroxybutyrate, acetone, and isopropyl alcohol show an inverse relationship with blood ethanol levels, though recent data has revealed that the antiketonemic effect of ethanol intake in hypothermia victims mainly influences acetone levels. Alternative biological samples including vitreous, urine, pericardial, and cerebrospinal fluids may be collected and analyzed for the determination of $\beta$-hydroxybutyrate, acetone, and isopropyl alcohol should blood be unavailable at autopsy [4, 24, 34, 35, 38-41].

It has been demonstrated that decompositional changes are not associated with $\beta$-hydroxybutyrate production. Furthermore, blood $\beta$-hydroxybutyrate levels in decomposed bodies can be considered an appropriate biochemical parameter in the estimation of $\beta$-hydroxybutyrate concentrations at the time of death. Indeed, $\beta$-hydroxybutyrate concentration does not increase after death and may, at most, decrease as a result of spontaneous molecule degradation [41-43].

Recent studies have indicated that $\beta$-hydroxybutyrate can be reliably measured in liver homogenates and may prove useful in confirming the presence of metabolic disturbances at the time of death. Liver homogenates can therefore be considered an alternative biological sample for $\beta$ hydroxybutyrate determination; limited amounts of biological fluids must be reserved for toxicology and biochemical analyses [43].

Normal levels of blood $\beta$-hydroxybutyrate, acetone, and isopropyl alcohol in suspected hypothermia fatalities do not allow this diagnosis to be excluded, particularly in cases with increased blood levels of ethanol. Furthermore, the severity of the metabolic disturbances present at the time of death cannot be evaluated based on levels of vitreous, urine, pericardial, or cerebrospinal fluid alone. Indeed, the blood-vitreous, bloodpericardial fluid, and blood-cerebrospinal fluid equilibrium is established following different time courses. Rapid increases in blood $\beta$-hydroxybutyrate and acetone levels cannot be reflected in simultaneous increases in the concentrations of vitreous, pericardial, and cerebrospinal fluid $\beta$ hydroxybutyrate and acetone. An even more careful interpretation is required when evaluating concentrations of urine $\beta$ hydroxybutyrate and acetone, since urine production in the kidneys follows different mechanisms from those of vitreous, pericardial, and cerebrospinal fluid production [4, 39, 40, 42].

Urine catecholamine, blood free fatty acids, blood corticosteroids (especially cortisol), and urine free cortisol may be increased in hypothermia fatalities, irrespective of blood ethanol concentrations. Though their determination may provide further information to confirm the diagnosis of death by hypothermia, preservation measures during sample collection and time after death may significantly influence catecholamine stability in urine. Thus, normal levels of urine adrenaline in suspected hypothermia fatalities do not allow this diagnosis to be excluded. Similarly, increases in blood free fatty acids and corticosteroid levels cannot be regarded as the sole criterion for the diagnosis of fatal hypothermia, and likewise, normal blood levels of these molecules do not allow this diagnosis to be ruled out. Increased and decreased corticosteroid levels in both blood and urine can also be the 
expression of preexisting diseases, leading to the conclusion that glucocorticosteroids, like other biochemical parameters, may be treated as potential markers of fatal hypothermia in the context of all postmortem investigation results $[24,34,35,37,44]$.

\section{Postmortem imaging and molecular pathology}

Postmortem imaging and molecular pathology are relatively more recent fields of research in the forensic setting and have shown encouraging results pertaining to the diagnosis of hypothermia [45-51]. Aghayev et al. [45] observed that postmortem magnetic resonance imaging (MRI) allowed iliopsoas and back muscle hemorrhages to be easily identified, whereas Wischnewski spots remained undetected. Michiue et al. [46] investigated postmortem lung air distribution in forensic autopsy cases using computed tomography (CT) and observed that hypothermia was characterized by diffuse hyperaeration with decreased vascularity, indicative of diffuse pulmonary emphysema and increased airway dead space. The authors postulated that these results could be the consequence of respiratory center impairment occurring in the process of death. This same radiologic pattern was nonetheless observed in obstructive pulmonary disease and starvation, suggesting that alternative pathophysiologic mechanisms may explain radiological increased airway dead space and limited hypostatic opacification (such as reduced pulmonary blood flow caused by alveolar overinflation in obstructive pulmonary disease and terminal hyperventilation due to acidosis in starvation). Kawasumi et al. [47] observed that many hypothermic deaths presented urine retention in the bladder (probably reflecting the effect of cold diuresis) on postmortem CT. Additional observations included blood clotting in the heart, pulmonary artery, or thoracic aorta (possibly indicating prolonged death process) and the lack of an increase in lungfield concentration (suggesting underlying dried lungs). Lastly, Hyodoh et al. [48] investigated postmortem lung air distribution in forensic autopsy cases using $\mathrm{CT}$ and found that hypothermia cases had higher aerated lung volumes and higher percentages of aerated lung volume compared to control cases. Based on these findings, the authors postulated that hypothermia fatalities were characterized by preserved pulmonary aeration and less important pulmonary hypostasis.

Investigations in molecular pathology performed in study populations including hypothermia fatalities revealed high messenger RNA (mRNA) expression levels of pulmonary surfactant-associated proteins A and D (SP-A1b, SP-A2b, and SP-D), increased mRNA expression levels of intrapulmonary matrix metalloproteinase-9 (suggesting partial damage of extracellular matrix), and high mRNA expression levels of atrial and brain natriuretic peptides in both left and right atrial and ventricular walls, possibly indicating persistent congestion and heart failure without substantial myocardial damage during cold exposure [49-51].

\section{Conclusion}

Despite promising advances in several fields of forensic research in recent years, the postmortem diagnosis of fatal hypothermia remains problematic. While the absence of supportive macroscopic, microscopic, and biochemical findings does not allow the diagnosis to be ruled out, their presence does not necessarily indicate it. Indeed, macroscopic and microscopic findings as well as biochemical results may vary considerably from case to case and may be influenced by numerous factors and circumstances, thus making them only a possible indication of hypothermia.

Diagnosis is particularly difficult in cases of acute hypothermia where physiological reactions against cold (e.g., hormonal release and biochemical response) could not be fully activated and death occurs before energy reserves (i.e., glycogen content) are utilized. In forensic practice, the most common factor accelerating cooling and shortening exposure time to cold (thus preventing macroscopic and microscopic hypothermia sign appearance) is alcohol intoxication.

The results of biochemical investigations are useful in detecting adaptation responses to cold stress as well as metabolic changes occurring after exposure to cold. Some parameters (blood ketones, cortisol, and free fatty acids as well as urine catecholamine and cortisol) can be considered reliable markers of fatal hypothermia. Nevertheless, abnormalities in biochemical results must be interpreted carefully, as they do not allow the diagnosis to be either categorically excluded or formally confirmed. Blood ethanol levels, postmortem interval, preservation measures during sampling as well as preexisting metabolic dysfunctions and endocrine disorders may all contribute to the increasing or decreasing of certain molecule concentrations. Simultaneous analyses focusing on a combination of biomarkers are therefore strongly recommended in order to reach appropriate conclusions.

Hypothermia may be responsible for the death of healthy people (either sober or under the influence of alcohol or other drugs) who can accidentally be exposed to cold temperatures in winter and are unable to obtain help. This scenario implies that hypothermia is the sole and exclusive cause of death and represents only a portion of the cases that may be encountered in forensic pathology routine. In these situations, the diagnosis remains essentially a medley of macroscopic, microscopic, and biochemical observations and can only be established per exclusionem by ruling out all other possible causes of death. Postmortem imaging, autopsy, histology, toxicology, and biochemistry are therefore mandatory.

However, most cases of forensic interest are much more nuanced. These cases pertain to subjects suffering from 
preexisting diseases (coronary atherosclerosis, respiratory disease, intracranial hemorrhage, chronic alcohol misuse, metabolic disturbances) or trauma (accidental or inflicted), or both, which can potentially be responsible for loss of consciousness, coma, and death on their own. When these subjects are found dead in environmental conditions possibly suggesting exposure to cold and postmortem investigations reveal also, though not exclusively, some of those findings supporting the diagnosis of hypothermia, the question of whether hypothermia is the main or only a contributing cause of death must be legitimately raised. Moreover, metabolic syndromes such as diabetic and alcoholic acidosis have typically negative autopsies and share specific biochemical features with hypothermia fatalities (i.e., increased blood ketones), rendering the identification of the cause(s) of death extremely challenging.

In these situations, the most appropriate medicolegal approach should allow preexisting diseases and trauma to be characterized as best as possible, in order to distinguish the ultimate cause of death from other contributing or secondary disorders. To do this, thorough, exhaustive, and complete medicolegal investigations including imaging, histology, immunohistochemistry, biochemistry, and toxicology are inevitably compulsory. Meticulous investigations at the death scene as well as the evidence of exposure to low environmental temperatures are of utmost importance for the accurate evaluation of all potentially hypothermia-related mortalities. All possible environmental risk factors for hypothermia should therefore be minutely sought out and documented.

Lastly, a multidomain approach focusing on a more integrated panel of postmortem investigations should be promoted among forensic pathologists. Such a multidisciplinary approach would allow more accurate diagnoses of hypothermia to be reached. In addition, some of the hypotheses formulated in (at present) isolated studies by distinct groups of authors might be validated, confirmed, or invalidated, thus contributing to a better understanding of the pathophysiological responses that take place during hypothermia-related deaths.

Acknowledgments The authors are grateful to the anonymous reviewers whose constructive and useful comments improved the quality of the article.

\section{References}

1. Soar J, Perkins GD, Abbas G, Alfonzo A, Barelli A, Bierens JJ, Brugger H, Deakin CD, Dunning J, Georgiou M, Handley AJ, Lockey DJ, Paal P, Sandroni C, Thies KC, Zideman DA, Nolan JP (2010) European Resuscitation Council Guidelines for Resuscitation 2010 Section 8. Cardiac arrest in special circumstances: electrolyte abnormalities, poisoning, drowning, accidental hypothermia, hyperthermia, asthma, anaphylaxis, cardiac surgery, trauma, pregnancy, electrocution. Resuscitation 81:1400-1433

2. Nixdorf-Miller A, Hunsaker DM, Hunsaker JC 3rd (2006) Hypothermia and hyperthermia medicolegal investigation of morbidity and mortality from exposure to environmental temperature extremes. Arch Pathol Lab Med 130:1297-1304

3. Lim C, Duflou J (2008) Hypothermia fatalities in a temperate climate: Sydney, Australia. Pathology 40:46-51

4. Teresiński G, Buszewicz G, Mądro R (2005) Biochemical background of ethanol-induced cold susceptibility. Leg Med (Tokyo) 7: $15-23$

5. Zachariah SB, Zachariah A, Ananda R, Stewart JT (2000) Hypothermia and thermoregulatory derangement induced by valproic acid. Neurology 55:150-151

6. Kreuzer P, Landgrebe M, Wittmann M, Schecklmann M, Poeppl TB, Hajak G, Langgut B (2012) Hypothermia associated with antipsychotic drug use: a clinical case series and review of current literature. J Clin Pharmacol 52:1090-1097

7. Türk EE (2010) Hypothermia. Forensic Sci Med Pathol 6:106-115

8. Dolinak D, Matshes E, Lew E (2005) Hypothermia. In: Forensic pathology. Principles and practice. Elsevier, Academic, 1st ed. San Diego pp 248-249

9. Madea B, Tsokos M, Preuss J (2008) Death due to hypothermia. Morphological findings, their pathogenesis and diagnostic value. In: Tsokos M (ed.), Forensic pathology reviews, vol. 5, pp 3-21

10. Hirvonen J (1976) Necropsy findings in fatal hypothermia cases. Forensic Sci 8:155-164

11. Coe JI (1984) Hypothermia: autopsy findings and vitreous glucose. J Forensic Sci 29:389-395

12. Hejna P, Zátopková L, Tsokos M (2012) The diagnostic value of synovial membrane hemorrhage and bloody discoloration of synovial fluid ("inner knee sign") in autopsy cases of fatal hypothermia. Int J Legal Med 126:415-419

13. Preuss J, Lignitz E, Dettmeyer R, Madea B (2007) Pancreatic changes in cases of death due to hypothermia. Forensic Sci Int 166:194198

14. Tsokos M, Rothschild MA, Madea B, Risse M, Sperhake JP (2006) Histological and immunohistochemical study of Wischnewsky spots in fatal hypothermia. Am J Forensic Med Pathol 27:70-74

15. Mizukami H, Shimizu K, Shiono H, Uezono T, Sasaki M (1999) Forensic diagnosis of death from cold. Leg Med 1:204-209

16. Türk EE, Sperhake JP, Madea B, Preuss J, Tsokos M (2006) Immunohistochemical detection of hemoglobin in frost erythema. Forensic Sci Int 158:131-134

17. Hirvonen J (2000) Some aspects on death in the cold and concomitant frostbites. Int J Circumpolar Heath 59:131-136

18. Ogata M, Ago K, Kondo T, Kasai K, Ishikawa T, Mikuzami H (2007) A fatal case of hypothermia associated with hemorrhages of the pectoralis minor, intercostals, and iliopsoas muscles. Am J Forensic Med Pathol 28:348-352

19. Nikolić S, Zivković V, Juković F, Babić D, Stanojkovski G (2009) Simon's bleedings: a possible mechanism of appearance and forensic importance - a prospective autopsy study. Int J Legal Med 123:293297

20. Zivković V, Nikolić S (2013) The unusual appearance of black esophagus in a case of fatal hypothermia: a possible underlying mechanism. Forensic Sci Med Pathol. doi:10.1007/s12024-0139445-3

21. Wolf DA, Aronson JF, Rajaraman S, Veasey SP 3rd (1999) Wischnewski ulcers and acute pancreatitis in two hospitalized patients with cirrhosis, portal vein thrombosis, and hypothermia. J Forensic Sci 44:1082-1085

22. Rothschild MA, Schneider V (1995) "Terminal burrowing behaviour"- a phenomenon of lethal hypothermia. Int J Legal Med 107: 250-256

23. Preuss J, Dettmeyer R, Lignitz E, Madea B (2004) Fatty degeneration in renal tubule epithelium in accidental hypothermia victims. Forensic Sci Int 141:131-135

24. Palmiere C, Mangin P (2013) Postmortem biochemical investigations in hypothermia fatalities. Int J Legal Med 127:267-276 
25. Preuss J, Dettmeyer R, Lignitz E, Madea B (2006) Fatty degeneration of myocardial cells as a sign of death due to hypothermia versus degenerative deposition of lipofuscin. Forensic Sci Int 159:1-5

26. Ishikawa T, Miyaishi S, Tachibana T, Ishizu H, Zhu BL, Maeda H (2004) Fatal hypothermia related vacuolation of hormone-producing cells in the anterior pituitary. Leg Med (Tokyo) 6:157-163

27. Doberentz E, Preuss-Wössner J, Kuchelmeister K, Madea B (2011) Histological examination of the pituitary glands in cases of fatal hypothermia. Forensic Sci Int 207:46-49

28. Ishikawa T, Quan L, Li DR, Zhao D, Michiue T, Hamel M, Maeda H (2008) Postmortem biochemistry and immunohistochemistry of adrenocorticotropic hormone with special regard to fatal hypothermia. Forensic Sci Int 179:147-151

29. Preuss J, Dettmeyer R, Poster S, Lignitz E, Madea B (2008) The expression of heat shock protein 70 in kidneys in cases of death due to hypothermia. Forensic Sci Int 176:248-252

30. Wang Q, Ishikawa T, Michiue T, Zhu BL, Guan DW, Maeda H (2012) Evaluation of human brain damage in fatalities due to extreme environmental temperature by quantification of basic fibroblast growth factor (bFGF), glial fibrillary acidic protein (GFAP), S100 $\beta$ and single-stranded DNA (ssDNA) immunoreactivities. Forensic Sci Int 219:259-264

31. Kitamura O, Gotohda T, Ishigami A, Tokunaga I, Kubo S, Nakasono I (2005) Effect of hypothermia on postmortem alterations in MAP2 immunostaining in the human hippocampus. Leg Med (Tokyo) 7: 340-344

32. Wang Q, Ishikawa T, Quan L, Zhao D, Li DR, Michiue T, Chen JH, Zhu BL, Maeda H (2009) Immunohistochemical distribution of basic fibroblast growth factor (bFGF) in medicolegal autopsy. Leg Med (Tokyo) 11(Suppl 1):S161-S164

33. Ishikawa T, Zhu BL, Li DR, Zhao D, Michiue T, Maeda H (2006) Age-dependent increase of clusterin in the human pituitary gland. Leg Med (Tokyo) 8:28-33

34. Bańka K, Teresiński G, Buszewicz, Mądro R (2013) Glucocorticosteroids as markers of death from hypothermia. Forensic Sci Int 229:60-65

35. Palmiere C, Bardy D, Letovanec I, Mangin P, Iglesias K, Augsburger M, Ventura F, Werner D (2013) Biochemical markers of fatal hypothermia. Forensic Sci Int 226:54-61

36. Teresiński G, Buszewicz G, Dąbrowski W, Mądro R (2007) Ketone bodies in controlled intraoperative hypothermia - preliminary study. Arch Med Sadowej Kryminol 57:389-393

37. Pakanen L, Kortelainen ML, Särkioja T, Porvari K (2011) Increased adrenaline to noradrenaline ratio is a superior indicator of antemortem hypothermia compared with separate catecholamine concentrations. J Forensic Sci 56:1213-1218
38. Teresiński G, Buszewicz G, Mądro R (2002) The influence of ethanol on the level of ketone bodies in hypothermia. Forensic Sci Int 127: $88-96$

39. Teresiński G, Buszewicz G, Mądro R (2009) Acetonaemia as an initial criterion of evaluation of a probable cause of sudden death. Leg Med (Tokyo) 11:18-24

40. Palmiere C, Sporkert F, Werner D, Bardy D, Augsburger M, Mangin P (2012) Blood, urine and vitreous isopropyl alcohol as biochemical markers in forensic investigations. Leg Med (Tokyo) 14:17-20

41. Buszewicz G, Teresiński G, Bańka K, Mądro R (2007) Diagnostic usefulness of the $\beta$-hydroxybutyrate/acetone ratio in medico-legal diagnostics of sudden death. Arch Med Sadowej Kryminol 57:289293

42. Palmiere C, Mangin P, Werner D (2014) Postmortem distribution of 3-beta-hydroxybutyrate. J Forensic Sci 59:161-166

43. Palmiere C, Mangin P, Werner D (2013) Preliminary results on the postmortem measurement of 3-beta-hydroxybutyrate in liver homogenates. Int J Legal Med 127:943-949

44. Bańka K, Teresiński G, Buszewicz G (2014) Free fatty acids as markers of death from hypothermia. Forensic Sci Int 234:79-85

45. Aghayev E, Thali MJ, Jackowski C, Sonnenschein M, Dirnhofer R, Yen K (2008) MRI detects hemorrhages in the muscles of the back in hypothermia. Forensic Sci Int 176:183-186

46. Michiue T, Sakurai T, Ishikawa T, Oritani S, Maeda H (2012) Quantitative analysis of pulmonary pathophysiology using postmortem computed tomography with regard to the cause of death. Forensic Sci Int 220:232-238

47. Kawasumi Y, Onozuka N, Kakizaki A, Usui A, Hosokai Y, Sato M, Saito H, Ishibashi T, Hayashizaki Y, Funayama M (2013) Hypothermic death: possibility of diagnosis by post-mortem computed tomography. Eur J Radiol 82:361-365

48. Hyodoh H, Watanabe S, Katada R, Hyodoh K, Matsumoto H (2013) Postmortem computed tomography lung findings in fatal of hypothermia. Forensic Sci Int 231:190-194

49. Miyazato T, Ishikawa T, Michiue T, Maeda H (2012) Molecular pathology of pulmonary surfactants and cytokines in drowning compared with other asphyxiation and fatal hypothermia. Int J Legal Med 126:581-587

50. Chen JH, Michiue T, Ishikawa T, Maeda H (2012) Molecular pathology of natriuretic peptides in the myocardium with special regard to fatal intoxication, hypothermia, and hyperthermia. Int J Legal Med 126:747-756

51. Wang Q, Ishikawa T, Michiue T, Zhu BL, Guan DW, Maeda H (2013) Molecular pathology of pulmonary edema in forensic autopsy cases with special regard to fatal hyperthermia and hypothermia. Forensic Sci Int 228:137-141 